\title{
Black CEsophagus as a Possible Complication of Cholangitis
}

\author{
Soumaya Jellal $^{1 *}$, Soukaina Jiddi ${ }^{2}$, Adil Ait Errami ${ }^{1}$, Sofia Oubaha ${ }^{2}$, Zouhour Samlani ${ }^{1}$ and Khadija Krati ${ }^{1}$
}

${ }^{1}$ Department of Gastroenterology, Mohammed VI University Hospital, Marrakech, Morocco

${ }^{2}$ Laboratory of Physiology, Cadi Ayyad University, Marrakech, Morocco

DOI: $10.36347 /$ simcr.2020.v08i03.041

| Received: 09.01.2020 | Accepted: 16.01.2020 | Published: 27.03.2020

*Corresponding author: Soumaya Jellal

Abstract

Acute esophageal necrosis (AEN), also known as black oesophagus or necrotizing esophagitis is a very rare clinical disorder characterized by a striking endoscopic image of diffuse, circumferential, black-appearing in the distal esophagus that stops abruptly at the gastroesophageal junction on esophagogastroduodenoscopy, its etiology is likely unclear and multifactorial, Upper gastrointestinal bleeding is The most common presentation, the incidence reported is only $0.2 \%$, the prognostic is poor and more the cases are fatal by comorbidities, We present a new case of acute oesophageal necrosis as a possible complication of cholangitis.

Keywords: Black œsophagus, Complication Cholangitis.

Copyright @ 2020: This is an open-access article distributed under the terms of the Creative Commons Attribution license which permits unrestricted use, distribution, and reproduction in any medium for non-commercial use (NonCommercial, or CC-BY-NC) provided the original author and source are credited.

\section{INTRODUCTION}

Acute esophageal necrosis (AEN), also known as black oesophagus or necrotizing esophagitis is a very rare clinical disorder characterized by a striking endoscopic image of diffuse, circumferential, blackappearing in the distal esophagus that stops abruptly at the gastroesophageal junction on esophagogastroduodenoscopy [1], its first described by Goldenburg and al. in 1990 [2] and later Gurvits classified AEN as a distinct syndrome in 2007, the incidence reported is only $0.2 \%$ [10].

its etiology is likely unclear and Various kinds of diseases are known to be associated with AEN. They include diabetes mellitus, malignancy, hypertension, chronic pulmonary disease, alcohol abuse, and trauma [3]. The physiopathology is multifactorial and most investigators suggested a combination of various mechanisms including ischemia, impaired mucosal barrier systems, and a backflow injury from gastric contents.

Upper gastrointestinal bleeding is the most common presentation including hematemesis, coffeegrounds emesis, melena, and blood loss anemia [5].

Complications may include stenosis or stricture formation in the distal esophagus, perforation, mediastinitis, and death. Overall mortality is largely related to the underlying medical condition and approaches $32 \%[1]$.
We present a new case of acute oesophageal necrosis as a possible complication of cholangitis

\section{Case Report}

A 59 years old man, without a past medical history, complained of epigastralgia for 5 months, At hospital he started to vomit and he had fever, admission blood pressure was 120/60physical, his pulse was regular with an apical rate of 104 beats/min, temperature $38.8^{\circ} \mathrm{C}$ examination epigastric sensitivity, his laboratory findings showed an increase in his inflammatory markers (CRP at 127 an white cells at 13000), A lipase test mesure was normal, Elevated cholestasis parameters, blood glucose of $1.2 \mathrm{~g} / \mathrm{dl}$, an increased level of serum creatinine $(49,4 \mathrm{~g} / \mathrm{l})$ and urea $(1.7 \mathrm{~g} / \mathrm{l})$ with hyponatremia $(122 \mathrm{mmol} / \mathrm{l})$, CT scan, performed to rule out a mass of $47 \mathrm{~mm}$ in the head of the pancreas with dilatation of the common bile duct and the main pancreatic duct, for suspected cholangitis due to bilary obstruction by the pancreatic tumour, patient was given Intravenous antibiotics, analgesia, antiemetics, IV proton inhibitor and fluids with restriction of oral intake.

One day after his admission, he developed coffee ground emesis, His hemoglobin dropped from 12.5 to $7.6 \mathrm{gm} / \mathrm{dl}$, and emergent esophagogastroduodenoscopy (EGD) was done which revealed diffusely circumferential black pigmentation of the oesophageal mucosa above the gastroesophageal 
junction with ulcerations of the distal thirds of the esophagus, biopsies were not taken, the patient died 2 days after the diagnosis.

\section{DISCUSSION}

Acute esophageal necrosis (AEN), also known as black oesophagus or necrotizing esophagitis is a very rare clinical disorder, First described by Goldenburg et al. in 1990 [2] and later Gurvits classified AEN as a distinct syndrome in 2007, the incidence is low, a 1 year prospective study of EDG have estimated the incidence at $0.2 \%$ [10], Gurvits in his review study have reported A total of 88 patients in the literature during the 40 years from 1965 to 2007 , it has a male predominance $(81 \%)$ with an average age of 67 years [4].

Its etiology is likely unclear and various kinds of diseases are known to be associated with AEN. They include diabetes mellitus, malignancy, hypertension, chronic pulmonary disease, alcohol abuse, trauma, systemic inflammatory response syndrome due to severe pancreatitis [3], and infections such as cytomegalovirus, Candida albicans and Klebsiellapneumoniae [11] have also been, Nasogastric intubation and endoscopic instrumentation with detergent use have been implicated as possible inciting events for the development of AEN [4]The physiopathology is multifactorial and most investigators suggested a combination of various mechanisms including ischemia, impaired mucosal barrier systems, and a backflow injury from gastric contents.

Most commonly reported reasons for admission were upper GI bleeding; other symptoms include abdominal pain, dysphagia, vomiting, epigastric pain, chest pain and a cardiovascular event or shock including tachycardia and hypotension [4].

AEN can be diagnosed endoscopically and is usually confirmed histologically; A biopsy is usually recommended but not required for a diagnosis of AEN.

The classic endoscpic appearance is a Circumferentially black appearing esophageal mucosa that stops abruptly at the gastroesophageal junction [4], other endoscopic findings may include gastric ulceration, dilated fluid-filled stomach, volvulus, gastric outlet obstruction and duodenitis or pyloric stenosis $[12,4]$.

There is no specific treatment, Development of AEN carries a generally poor prognosis and the goal of therapy should be directed at treating the coexisting medical diseases.

The majority of authors support a convervating approach consisting on intravenous hydratation, blood cell transfusion, Intravebous proton pump inhibitor, supportive parenteral alimentation and correcting the underlying disease, nasogastric tube inserstion is contraindicated due to risk of perforation $[4,1]$.

The prognosis of AEN is poor, mortality is high $(31.8 \%)$ and is probably related to the associated conditions and secondary to complications as esophageal perforation and mediastinal infection [4].

After our literature analysis we did not find any case of AEN associated with cholangitits which make our case unique

\section{REFERENCES}

1. Black esophagus: Acute esophageal necrosis syndrome Grigoriy E Gurvits World J Gastroenterol. 2010 Jul 14; 16(26): 32193225. Published online 2010 Jul 14.

2. Acute necrotizing esophagitis. Goldenberg SP, Wain SL, Marignani P. Gastroenterology. 1990; 98:493-6.

3. Yamamoto T, Miura A, Nishio H. Autopsy case of acute esophageal necrosis associated with fatal diabetic ketoacidosis. Romanian Journal of Legal Medicine. 2018 Jun 1;26(2):134-7.

4. Gurvits GE, Shapsis A, Lau N, Gualtieri N, Robilotti JG. Acute esophageal necrosis: a rare syndrome. Journal of gastroenterology. 2007 Jan 1;42(1):29.

5. Marinucci C, Zardo F, Musso A, Strignano P, di Cella SM, Porta M. A severe case of epigastric pain, diarrhea and coffee ground vomitus. Internal and emergency medicine. 2018 Oct 1;13(7):1097101.

6. Mealiea D, Greenhouse D, Velez M, Moses P, Marroquin CE. Case Report: Acute Esophageal Necrosis In An Immunosuppressed KidneyTransplant Recipient.

7. Iqbal S, Leong MH. Acute esophageal necrosis: a case series and its management. Journal of surgical case reports. 2018 Dec 12;2018(12):rjy328.

8. Acute Esophageal Necrosis: A View in the Dark Khan Case Rep Gastroenterol. 2019;13:25-31

9. Averbukh LD, Mavilia MG, Gurvits GE. Acute esophageal necrosis: a case series. Cureus. 2018 Mar;10(3).

10. Soussan EB, Savoye G, Hochain P, Hervé S, Antonietti M, Lemoine F, Ducrotté P. Acute esophageal necrosis: a 1-year prospective study. Gastrointestinal endoscopy. 2002 Aug 1;56(2):2137.

11. Liu YH, Lin YS, Chen HJ, Tu CY, Chen W. Klebsiella pneumoniae deep neck infection with acute necrotizing esophagitis. Southern medical journal. $2009 \mathrm{Feb}$;102(2):219-.

12. Reichart M, Busch OR, Bruno MJ, Van Lanschot JJ. Black esophagus: a view in the dark. Diseases of the Esophagus. 2000 Dec 1;13(4):311-3. 